\title{
Impaired NO-cGMP signaling in the pathogenesis of neonatal pulmonary hypertension and therapeutic implications
}

Steve H Abman

Address: Department of Pediatrics, Pediatric Heart Lung Center, University of Colorado School of Medicine, Aurora CO, USA

Email: Steve H Abman - steven.abman@ucdenver.edu

from 4th International Conference of cGMP Generators, Effectors and Therapeutic Implications

Regensburg, Germany. 19-21 June 2009

Published: II August 2009

BMC Pharmacology 2009, 9(SuppI I):SI doi:I0.1/86/I47I-22I0-9-SI-SI

This abstract is available from: http://www.biomedcentral.com/I47I-22I0/9/SI/SI

(C) 2009 Abman; licensee BioMed Central Ltd.

\section{Background}

Postnatal survival is dependent upon successful transition of the pulmonary circulation at birth. Persistent pulmonary hypertension of the newborn (PPHN) is a clinical syndrome that is characterized by the failure to achieve or sustain a marked reduction of pulmonary vascular resistance (PVR) after delivery. High PVR causes extra-pulmonary right-to-left shunt, leading to profound hypoxemia, asphyxia and significant morbidity and mortality. Past studies have shown that NO-cGMP signaling contributes significantly to the normal fall in PVR at birth, and that decreased NO production or activity contributes to PPHN. Multicenter studies have shown that inhaled NO is effective in the treatment of clinical PPHN, but nearly $40 \%$ of newborns with PPHN fail to respond to iNO therapy. These findings suggest that additional therapies are essential for enhancing NO-cGMP signaling and improving outcomes of neonates with severe PPHN. Since recent studies demonstrated increased oxidative stress in experimental PPHN, we hypothesized that impaired NO responsiveness in PPHN may be due to decreased soluble guanylate cyclase (sGC) activity due to oxidation. Cinaciguat (BAY 58-2667) is a novel sGC activator that causes vasodilation even in the presence of oxidized heme, but its hemodynamic effects in the perinatal lung are unknown. Therefore, we performed a series of physiologic studies in chronically-prepared, late gestation fetal lambs. Additional in vitro studies were performed with isolated pulmonary artery smooth muscle cells (SMC) from normal and PPHN lambs.

\section{Results}

We found that in the normal fetus, cinaciguat ( 0.1 to 100 mcg over $10 \mathrm{~min}$ ) caused dose-related increases in pulmonary blood flow greater than 4 -fold above baseline values and reduced PVR by $80 \%$. Treatment with ODQ, an sGC oxidizing inhibitor, enhanced cinaciguat-induced pulmonary vasodilation by over $150 \%$. The pulmonary vasodilator effect of cinaciguat was prolonged, decreasing PVR for more than 1.5 hours after brief intrapulmonary infusion. In vitro stimulation of fetal pulmonary artery SMC with cinaciguat after ODQ treatment demonstrated a 14 -fold increase in CGMP in comparison without ODQ. We further studied the pulmonary vascular effects of cinaciguat in fetal sheep with chronic pulmonary hypertension after partial ligation of the ductus arteriosus (PPHN). We found that in contrast with the loss of acetylcholineinduced pulmonary vasodilation 5 days after surgery, the cinaciguat response persisted and was enhanced in PPHN lambs. After cesarean-section delivery and mechanical ventilation, neonatal lambs with PPHN demonstrated markedly impaired vasodiliation to inhaled NO (20 and $40 \mathrm{ppm})$. In contrast, brief infusion of cinaciguat caused marked and prolonged pulmonary vasodilation.

\section{Conclusion}

Overall, these findings suggest that oxidized sGC contributes to the pathophysiology of PPHN, and that treatment with cinaciguat, an sGC activator, may provide a novel strategy in refractory PPHN. 